\title{
Preventing Surgical Site Infections: Is It Just Too Sweet? Reply
}

\author{
Michele Diana $\cdot$ Martin Hübner • \\ Marie-Christine Eisenring • Giorgio Zanetti • \\ Nicolas Troillet $\cdot$ Nicolas Demartines
}

Published online: 13 April 2011

(C) Société Internationale de Chirurgie 2011

We thank Dr. Ng and coworkers [1] for their interest in our article and for the insights about the importance of nutritional screening as a way to prevent surgical site infections (SSIs). We totally agree with the suggestion of $\mathrm{Ng}$ et al. that nutritional counseling should be completed with screening for early detection of insulin resistance before major digestive surgery.

Overt diabetes is strongly associated with a higher rate of SSIs [2]. The hyperglycemic postsurgical stress response has been associated with an increased risk of SSIs in nondiabetic patients also [3]. Intensive postoperative glycemia control has been shown to improve outcomes in nondiabetic cardiovascular patients [4], general surgery patients [5], and critically ill patients [6]. We could speculate that patients with metabolic syndrome and insulin resistance might probably benefit from a stringent protocol of glycemia control initiated in the early postoperative phase. As outlined in the recent Cochrane Review [7], there is insufficient evidence to support the use of strict glycemic

M. Diana $\cdot$ M. Hübner $(\bowtie) \cdot$ N. Demartines

Department of Visceral Surgery, University Hospital, Lausanne Medical School, Rue du Bugnon 46,

1011 Lausanne, Switzerland

e-mail: martin.hubner@chuv.ch

G. Zanetti $\cdot$ N. Troillet

Service of Hospital Preventive Medicine and Service

of Infectious Diseases, University Hospital,

Lausanne Medical School, Lausanne, Switzerland

M.-C. Eisenring · N. Troillet

Service of Infectious Diseases,

Central Institute of the Valais Hospitals,

Sion, Switzerland control in the intra- and postoperative periods for the prevention of SSIs. Nevertheless, it is our current practice to apply strict glycemic control to all patients who spend the first $24-48 \mathrm{~h}$ in the ICU after major operations (e.g., esophagectomy, pancreatic resection, and major hepatectomy).

Our survey has shown an overall low adherence of surgeons to using measures to prevent SSIs irrespective of the level of scientific evidence. Thus, should a preoperative screening for insulin resistance be part of the presurgery workup by the operating surgeon or should it be the role of the general practitioner taking care of the patient?

The lack of stringent evidence about the protective role of early detection of metabolic syndrome and other factors specifically connected to health-care reimbursement policies might hamper its implementation. Prospective assessment of the cost effectiveness of a systematic presurgery insulin resistance workup and systematic glycemic control on the SSI rate could be a valuable tool, provided that well-known risk factors and confounders are controlled.

Today's surgical practice is mainly developed outside the OR to improve quality of care and offer patients a tailored postoperative management. Enhanced surgical community attention to preoperative nutritional status assessment is a rather difficult task but one that we consider worthy. We recently published a meta-analysis on the role played by immunonutrition on the postoperative outcomes, and results favor the liberal use of this measure [8]. Furthermore, a prospective randomized clinical trial on the effects of immunonutrition on SSIs is currently ongoing at our General and Visceral Surgery Department.

To conclude, although blood sweetness appears deleterious for the surgical patient, it would, without doubt, be sweet to reduce SSI rates. 


\section{References}

1. Ng CSH, Wong RHL, Underwood MJ (2011) Preventing surgical site infections: is it just too sweet? World J Surg. doi:10.1007/ s00268-011-1006-x

2. Guvener M, Pasaoglu I, Demircin M, Oc M (2002) Perioperative hyperglycemia is a strong correlate of postoperative infection in type II diabetic patients after coronary artery bypass grafting. Endocr J 49(5):531-537

3. Ramos M, Khalpey Z, Lipsitz S, Steinberg J, Panizales MT, Zinner M, Rogers SO (2008) Relationship of perioperative hyperglycemia and postoperative infections in patients who undergo general and vascular surgery. Ann Surg 248(4):585-591

4. Swenne CL, Lindholm C, Borowiec J, Schnell AE, Carlsson M (2005) Peri-operative glucose control and development of surgical wound infections in patients undergoing coronary artery bypass graft. J Hosp Infect 61(3):201-212

5. Ata A, Lee J, Bestle SL, Desemone J, Stain SC (2010) Postoperative hyperglycemia and surgical site infection in general surgery patients. Arch Surg 145(9):858-864

6. van den Berghe G, Wouters P, Weekers F, Verwaest C, Bruyninckx F, Schetz M, Vlasselaers D, Ferdinande P, Lauwers P, Bouillon R (2001) Intensive insulin therapy in the critically ill patients. N Engl J Med 345(19):1359-1367

7. Kao LS, Meeks D, Moyer VA, Lally KP (2009) Peri-operative glycaemic control regimens for preventing surgical site infections in adults. Cochrane Database Syst Rev 2009(3):CD006806

8. Cerantola Y, Hubner M, Grass F, Demartines N, Schafer M (2011) Immunonutrition in gastrointestinal surgery. Br J Surg 98(1):37-48 